\title{
Synchronization of radiation in an oversized coaxial Ka-band backward wave oscillator using two-dimensional Bragg structure
}

\author{
N. S. Ginzburg, ${ }^{1,2}$ E. V. Ilyakov, ${ }^{1}$ I. S. Kulagin, ${ }^{1}$ N. Yu. Peskov, ${ }^{1}$ R. M. Rozental, ${ }^{1}$ \\ A. S. Sergeev, ${ }^{1}$ V. Yu. Zaslavsky, ${ }^{1,2}$ and I. V. Zheleznov ${ }^{1}$ \\ ${ }^{1}$ Institute of Applied Physics, Russian Academy of Sciences, Nizhny Novgorod, 603950 Russia \\ ${ }^{2}$ Nizhny Novgorod State University, Nizhny Novgorod, 603950 Russia
}

(Received 3 April 2015; published 11 December 2015)

\begin{abstract}
A coaxial Ka-band backward wave oscillator with a two-dimensional Bragg structure located at the output of the interaction space has been studied. This structure has a double-period corrugation and provides azimuthal electromagnetic energy fluxes, which act on the synchronized radiation of an oversized tubular electron beam. Proof-of-principle experiments were conducted based on the Saturn thermionic accelerator (300 keV/200 A/2 $\mu \mathrm{s})$. In accordance with simulations, narrow-band generation was obtained at a frequency of $30 \mathrm{GHz}$ and a power level of 1.5-2 MW. As a result, the possibility of using a twodimensional distributed feedback mechanism in oscillators of the Cherenkov type has been demonstrated.
\end{abstract}

DOI: 10.1103/PhysRevSTAB.18.120701

PACS numbers: 41.75. Ht, 52.35.Hr, 84.40.Fe

\section{INTRODUCTION}

By now, a radiation power of the gigawatt level was achieved in the $\mathrm{x}$-band oscillators based on high-current relativistic electron beams (REBs), such as relativistic backward wave oscillators (BWOs) and traveling wave tubes (TWTs) of different types [1-8]. The operating principle of these devices is based on the interaction of a rectilinear electron beam with rf field, which is slowed down by a metal structure with a periodic corrugation (slow-wave structure), and they are usually called Cherenkov devices or Smith-Purcell devices (see, e.g., [7]). The further increase in the radiated power of such oscillators would require an increase in the transverse size of the interaction space. Obviously, the use of oversized microwave systems is also needed for advancement of high-power Cherenkov devices into the millimeter and submillimeter wavelength bands to reduce Ohmic losses, as well as for transportation of intense REBs. One of the main problems of this way is provision of transverse mode selection in a strongly oversized system, which is necessary for the spatial coherence of radiation.

To solve this problem in free-electron masers (FEMs) driven by spatially extended REBs of sheet and tubular geometry, the use of two-dimensional (2D) distributed feedback was proposed $[9,10]$. Such a feedback can be realized on the basis of planar and coaxial 2D Bragg resonators having double-period corrugations. Experimental studies of the FEMs exploiting a novel feedback mechanism were performed in the $\mathrm{W}$ band (planar geometry) at the Budker Institute of Nuclear Physics (Novosibirsk, Russia) [11,12] and in the Ka band (coaxial

Published by the American Physical Society under the terms of the Creative Commons Attribution 3.0 License. Further distribution of this work must maintain attribution to the author(s) and the published article's title, journal citation, and DOI. geometry) at the University of Strathclyde (Glasgow, UK) [12-14] in collaboration with the Institute of Applied Physics RAS and FZK (Karlsruhe, Germany).

Successful FEM experiments encourage the implementation of a 2D distributed feedback mechanism in highpower relativistic oscillators of other types, including Cherenkov devices [15-18]. FEMs are based on stimulated emission of wiggling relativistic electrons and exploit the Doppler frequency up-shift. As a result, such devices are driven usually by relativistic beams having a fairly high energy $(0.5-1 \mathrm{MeV})$, which are generated by high voltage and rather bulky accelerators. Cherenkov masers are driven by rectilinear electron beams, which significantly simplify the electron-optical system and make it possible to employ more intensive REBs in comparison with FEMs. Cherenkov devices are less sensitive to the initial spread of beam parameters and can effectively operate in short $\mathrm{mm}$ and sub-mm bands, being driven by moderately relativistic (about $300 \mathrm{keV}$ ) electron beams. Thus, Cherenkov masers can be more compact, which is important for potential applications.

The present paper is devoted to theoretical and experimental studies of a Cherenkov backward wave oscillator driven by a large-size tubular REB. Coaxial geometry of the oscillator makes it possible to increase the total beam current and the output rf power while keeping the current and radiation densities constant. The use of an external 2D Bragg structure is proposed to realize 2D distributed feedback and to provide the spatial coherence of radiation in a strongly oversized microwave system [15].

\section{OPERATION PRINCIPLES AND SIMULATIONS OF A CHERENKOV BWO WITH EXTERNAL 2D BRAGG SYNCHRONIZER}

Schematic drawing of the coaxial BWO with an external 2D Bragg synchronizer is shown in Fig. 1(a). We assume 
that the coaxial waveguide has a small curvature of the surface, i.e., that the mean radius $r_{0}$ greatly exceeds the distance between the conductors $a_{0}$ and the radiation wavelength $\lambda$ :

$$
r_{0} \gg a_{0}, \quad r_{0} \gg \lambda .
$$

In this assumption, we can adopt a planar model $[19,20]$ and introduce the transverse coordinates $y=r$ and $x=r_{0} \varphi$ ( $r$ and $\varphi$ are the radial and azimuthal coordinates, respectively). We present the radiation field inside the waveguide in the form of two partial quasi-optical wave beams

$$
\vec{E}=\operatorname{Re}\left\{\vec{E}_{\mathrm{A}}^{0}(y)\left[\mathbf{A}_{+}(z, x, t) \mathrm{e}^{-\mathrm{i} h_{z} z}+\mathbf{A}_{-}(z, x, t) \mathrm{e}^{\mathrm{i} h_{z} z}\right] \mathrm{e}^{\mathrm{i} \omega t}\right\}
$$

of frequency $\omega$ and longitudinal wave numbers $\pm h_{\mathrm{z}}$ propagating in the forward $\left(\mathbf{A}_{+}\right)$and backward ( $\left.\mathbf{A}_{-}\right)$ directions with respect to the electron beam, where $\mathbf{A}_{ \pm}(x, z, t)$ are slow functions of the $x$ and $z$ coordinates and time, and the function $\vec{E}_{\mathrm{A}}^{0}(y)$ describes the radial distributions of the fundamental harmonic. Under condition (1), this structure coincides with the TM polarized mode of a planar waveguide and can be represented as

$$
\vec{E}_{\mathrm{A}}^{0}(y)=g_{0}^{2} \Psi \vec{z}_{0}-i h_{z} \frac{\partial \Psi}{\partial y} \vec{y}_{0}
$$

where $\Psi(y, z)=g_{0}^{-1} \sin \left(g_{0} y\right), g_{0}=n \pi / a_{0}$ is the transverse wave number of the fundamental harmonic, and the mode index $n$ is transverse over $y$ (radial).

The electron beam focused by the guide magnetic field moves in the $+z$ direction with the axial velocity $v_{\|}=\beta_{\|} c$. The interaction space is formed by a coaxial waveguide where both the inner and outer conductors have an antiphase azimuthally symmetric corrugation,

$$
r=r_{\mathrm{sw}} \cos \left(h_{\mathrm{sw}} z\right) .
$$

Here, $h_{\mathrm{sw}}=2 \pi / d_{\mathrm{sw}}$, where $d_{\mathrm{sw}}$ is the period of the slowwave structure [section 3 in Fig. 1(a) with the corrugation shown at the inner conductor]. Under the synchronism condition

$$
\omega \approx\left(h_{\mathrm{sw}}-h_{z}\right) v_{\|}
$$

(which can be considered as a counterpart of the Cherenkov synchronism in a dielectric structure), the rectilinear electron beam effectively interacts with the longitudinal field of the $(-1)$ spatial harmonic

$$
E_{z}=\operatorname{Re}\left[\mathbf{A}_{-}(z, x, t) E_{z}^{(-1)}(y) \mathrm{e}^{\mathrm{i} \omega t+\mathrm{i}\left(h_{z}-h_{s w}\right) z}\right],
$$

where
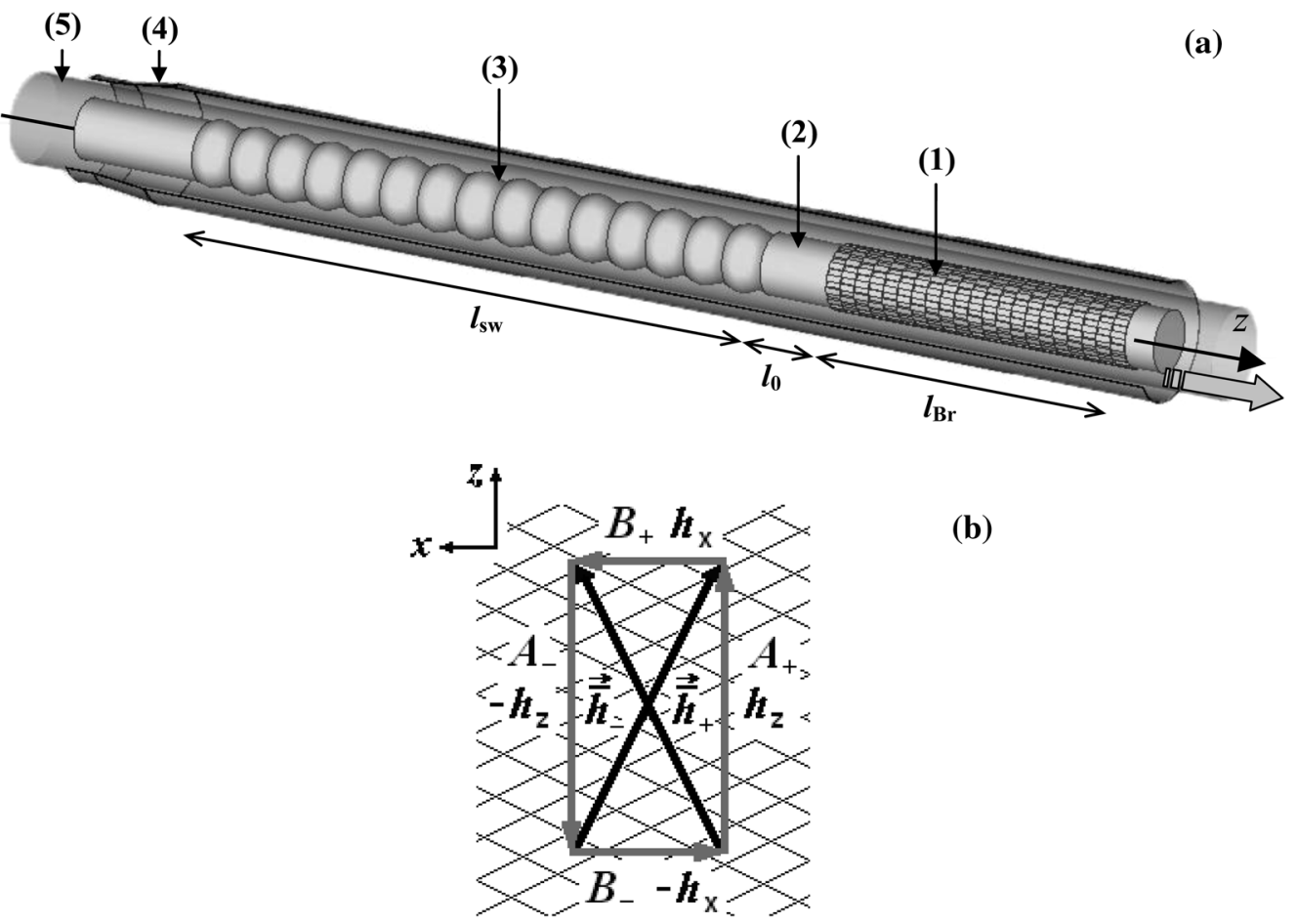

(b)

FIG. 1. (a) Scheme of the coaxial BWO with (1) external 2D Bragg synchronizer, (2) regular waveguide section, (3) slow-wave structure, (4) cutoff narrowing, (5) tubular electron beam and (b) the diagram illustrating scattering of the partial waves by a 2D Bragg structure $\left(\overrightarrow{\bar{h}}_{ \pm}=\bar{h}_{z} \vec{z}_{0} \pm \bar{h}_{x} \vec{x}_{0}\right.$ are the gratings vectors). 


$$
\begin{aligned}
E_{z}^{(-1)}(y)= & r_{\mathrm{sw}}\left(g_{0}^{2}+h_{z} h_{\mathrm{sw}}\right)\left\{\operatorname{sh}\left[g_{-1}\left(y-a_{0}\right)\right]\right. \\
& \left.+\operatorname{sh}\left(g_{-1} y\right)\right\} / 2 \operatorname{sh}\left(g_{-1} a_{0}\right)
\end{aligned}
$$

$h_{-1}=h_{z}-h_{\mathrm{sw}}$ and $g_{-1}=\sqrt{h_{-1}^{2}-k^{2}}$ are the longitudinal and transverse wave numbers of this harmonic, respectively, and $k=\omega / c$. In the quasioptical approximation, taking into account wave diffraction in the transverse (azimuthal) direction, this interaction can be described by the following equations:

$$
\begin{aligned}
\frac{\mathrm{i} C}{2} \frac{\partial^{2} A_{-}}{\partial X^{2}}+\left(\frac{\partial}{\partial \tau}-\frac{\partial}{\partial Z}\right) A_{-} & =\frac{1}{\pi} \int_{0}^{2 \pi} \mathrm{e}^{-\mathrm{i} \theta} \mathrm{d} \theta_{0} \\
\left(\frac{1}{\beta_{\|}} \frac{\partial}{\partial \tau}+\frac{\partial}{\partial Z}\right)^{2} \theta & =\operatorname{Re}\left(A_{-} \mathrm{e}^{\mathrm{i} \theta}\right)
\end{aligned}
$$

with the initial conditions

$$
\begin{gathered}
\left.A_{-}\right|_{\tau=0}=A_{0}(X, Z),\left.\quad \theta\right|_{Z=0}=\theta_{0} \in[0,2 \pi), \\
\left.\left(\frac{1}{\beta_{\|}} \frac{\partial}{\partial \tau}+\frac{\partial}{\partial Z}\right) \theta\right|_{Z=0}=\Delta .
\end{gathered}
$$

In Eqs. (5) and (6), we used the following normalized variables and parameters: $\tau=C \bar{\omega} t, X=C k x, Z=C k z$, $A_{-}=\mu e \mathrm{~A}_{-} / m c \bar{\omega} \gamma_{0}^{2} C^{2}, C=\sqrt[3]{\mu e I_{0} \Pi / m c^{2} \gamma_{0}}$ is the Pierce parameter, $I_{0}$ is the linear current density, $\mu=1 / \gamma_{0}^{2} \beta_{0}^{3}$ is the bunching parameter of electrons, $\gamma$ is their relativistic mass factor (the index " 0 " corresponds to the unperturbed values at the entrance to the interaction space), $\Pi=$ $8 \pi\left|E_{z}^{-1}\left(y_{\text {beam }}\right)\right|^{2} / a_{0} \bar{\omega} h_{z}\left(h_{z}-h_{\text {sw }}\right)^{2}$ is the electron-wave coupling coefficient (impedance), $\theta=\bar{\omega} t+\left(h_{z}-h_{\mathrm{sw}}\right) z$ is the electron phase with respect to the synchronous harmonic of the wave, and $\Delta=\left(\bar{\omega}+h_{z} v_{\|}-h_{\mathrm{sw}} v_{\|}\right) / \bar{\omega} C$ is the mismatch of synchronism at the carrier frequency. We consider the Bragg frequency $\bar{\omega}$ [see Eq. (12) below] as such a frequency, $\omega=\bar{\omega}$. We also note that in the electron motion equation $(5 b)$ we assume small changes in the electron energies. One more important remark is that the azimuthal diffraction of the wave beam described by the first term on the left-hand side of Eq. (5a) is an important factor that provides partial synchronization of radiation in the absence of the external 2D Bragg structure for the limited Fresnel parameter $F=l_{x}^{2} / \lambda l_{z} \leq 1$.

The up-stream cutoff narrowing [section 4 in Fig. 1(a)] reflects the backward propagating partial wave $\mathbf{A}_{-}$into the forward wave $\mathbf{A}_{+}$:

$$
\left.A_{+}\right|_{Z=0}=R A_{-}(X, \tau),
$$

which freely propagates in the $+z$ direction without interaction with the electrons over the interaction space.
In the normalization given above, the spatiotemporal evolution of this wave can be described as

$$
\frac{\mathrm{i} C}{2} \frac{\partial^{2} A_{+}}{\partial X^{2}}+\left(\frac{\partial}{\partial \tau}+\frac{\partial}{\partial Z}\right) A_{+}=0 .
$$

The 2D Bragg structure [section 1 in Fig. 1(a)] in the form of a coaxial waveguide section with double-period 2D corrugation,

$$
r=\frac{r_{1}}{4}\left[\cos \left(\bar{h}_{z} z-\bar{h}_{x} x\right)+\cos \left(\bar{h}_{z} z+\bar{h}_{x} x\right)\right],
$$

is located on the collector side of the device (we assume that only one conductor is corrugated). Here, $r_{1}$ is the corrugation depth, $\bar{h}_{z}=2 \pi / d_{z}$, where $d_{z}$ is the corrugation period over the longitudinal $z$ coordinate, and $\bar{h}_{x}=\bar{m} / r_{0}$, where $\bar{m}$ is the number of corrugation turns over the azimuth. Under the Bragg resonance condition

$$
h_{x}=\bar{h}_{x}, \quad h_{z}=\bar{h}_{z}
$$

( $h_{x}=M / r_{0}$, where $M$ is the azimuthal mode number), this structure provides coupling and mutual scattering of the four partial waves [Fig. 1(b)], namely, two axially propagating waves (2) (i.e., the forward wave $\mathbf{A}_{+}$and the backward wave $\mathbf{A}_{-}$) and two transversely propagating waves $\mathbf{B}_{ \pm}$of TE polarization (in the classification of eigenwaves of a coaxial waveguide)

$$
\vec{E}=\operatorname{Re}\left\{\vec{E}_{\mathrm{B}}^{0}(y)\left[\mathbf{B}_{+}(z, x, t) \mathrm{e}^{-\mathrm{i} h_{x} x}+\mathbf{B}_{-}(z, x, t) \mathrm{e}^{\mathrm{i} h_{x} x}\right] \mathrm{e}^{\mathrm{i} \bar{\omega} t}\right\} .
$$

Here and above,

$$
\bar{\omega}=c \sqrt{\bar{h}_{z}^{2}+g_{0}^{2}}=c \sqrt{\bar{h}_{x}^{2}+g_{0}^{2}}
$$

is the frequency of exact Bragg resonance. The azimuthal electromagnetic (e.m.) fluxes $\mathbf{B}_{ \pm}$, which appear on the corrugation of such a type and are finally scattered into the backward wave $\mathbf{A}_{-}$, synchronize radiation from a large-size tubular electron beam. The process of mutual scattering of the four partial waves in the quasioptical approximation (with the diffraction effects taken into account) is described by the following system of equations (cf. $[9,10]$ ):

$$
\begin{aligned}
& \frac{\mathrm{i} C}{2} \frac{\partial^{2} A_{ \pm}}{\partial X^{2}}+\left(\frac{\partial}{\partial \tau} \pm \frac{\partial}{\partial Z}\right) A_{ \pm}+\mathrm{i} \alpha\left(B_{+}+B_{-}\right)=0, \\
& \frac{\mathrm{i} C}{2} \frac{\partial^{2} B_{ \pm}}{\partial Z^{2}}+\left(\frac{\partial}{\partial \tau} \pm \frac{\partial}{\partial X}\right) B_{ \pm}+\mathrm{i} \alpha\left(A_{+}+A_{-}\right)=0,
\end{aligned}
$$

where, as in Eqs. (5) and (6), normalizations are employed. The wave coupling coefficient for the partial waves of $\mathrm{TM}\left(\mathbf{A}_{ \pm}\right)$and TE $\left(\mathbf{B}_{ \pm}\right)$types (such a feedback loop is 
used in the experimental realization of the BWO described below) is given by

$$
\alpha \approx \frac{r_{1} \sqrt{h_{x} h_{z}}}{4 a_{0} k C} .
$$

The slow wave structure and a 2D Bragg synchronizer are separated by a regular waveguide section of length $L_{0}$, where the partial waves do not interact with the electron beam.

Due to the coaxial geometry of the cavity, the partial waves should satisfy the cyclic boundary conditions

$$
\begin{aligned}
& \mathbf{A}_{ \pm}\left(X+L_{x} ; Z ; \tau\right)=\mathbf{A}_{ \pm}(X ; Z ; \tau), \\
& \mathbf{B}_{ \pm}\left(X+L_{x} ; Z ; \tau\right)=\mathbf{B}_{ \pm}(X ; Z ; \tau),
\end{aligned}
$$

where $L_{x}=2 \pi k r_{0} C$ is the normalized mean resonator perimeter. Conditions (15) allow the solution of Eqs. (5), (8), and (13) to be represented as a Fourier series:

$$
\begin{aligned}
& A_{ \pm}(X ; Z ; \tau)=\sum_{m=-\infty}^{\infty} A_{ \pm}^{m}(Z ; \tau) \mathrm{e}^{\mathrm{i} s m X}, \\
& B_{ \pm}(X ; Z ; \tau)=\sum_{m=-\infty}^{\infty} B_{ \pm}^{m}(Z ; \tau) \mathrm{e}^{\mathrm{i} s m X},
\end{aligned}
$$

where $s=2 \pi / L_{x}$. Each Fourier term in Eq. (16) can be considered as an azimuthal mode. In this case, the azimuthal mode index for the waves $\mathbf{A}_{ \pm}$is $m$, while for the waves $\mathbf{B}_{ \pm}$it can be introduced as $M \pm m$.

Under the assumption that partial waves do not reflect from the ends of the corrugated surface and e.m. fluxes from the outside of the resonator are absent, the boundary conditions for Eq. (13) take the form

$$
\left.A_{-}\right|_{Z=L_{z}}=0
$$

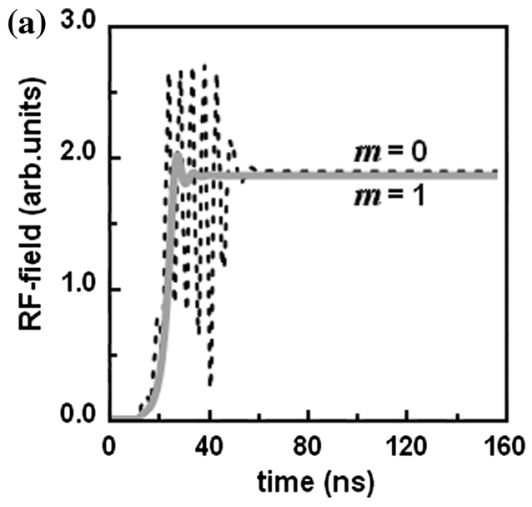

$$
B_{ \pm}^{m}-\sqrt{\frac{C}{2 \pi \mathrm{i}}} \int_{0}^{\tau} \frac{e^{\mp \mathrm{i} s m\left(\tau-\tau^{\prime}\right)}}{\sqrt{\tau-\tau^{\prime}}} \frac{\partial B_{ \pm}^{m}}{\partial Z} \mathrm{~d} \tau^{\prime}=\left.0\right|_{\substack{Z=L_{\mathrm{sw}}+L_{0} \\ Z=L_{z}}}
$$

where $L_{z}=L_{\mathrm{sw}}+L_{0}+L_{\mathrm{Br}}$ is the total normalized length of the system [see Fig. 1(a)]. Note that boundary conditions (17b) for the transversely propagating waves $\mathbf{B}_{ \pm}$are similar to the nonreflected boundary conditions which are widely used in gyrotron theory for quasicutoff modes. The procedure of deriving such conditions is described in detail in [21].

The simulations we carried out are aimed at studying the evolution of different operating regimes in the coaxial BWO when the transverse size of the interaction space is increased. The simulation parameters were close to the experimental realization described in Sec. III below. In the regime of "free oscillations" (BWO without the 2D Bragg structure), a single-mode operating regime is established in a cavity of moderate perimeter $l_{\mathrm{x}} / \lambda \approx 3-5$ (Fig. 2). However, the operating azimuthal mode in the stationary regime can be different and depends on initial conditions. An increase in the cavity perimeter up to $l_{\mathrm{x}} / \lambda \geq 10-6$ leads to multifrequency oscillations with the simultaneous excitation of different azimuthal modes $m=0, \pm 1, \pm 2 \ldots$. (Fig. 3, left column). Installation of an external 2D Bragg synchronizer into the cavity having such an oversize parameter results in onset of single-frequency oscillations at the azimuthally symmetric mode (Fig. 3, right column).

It should be noted that the planar model exploited in the simulations is valid for a strongly oversized system. Nevertheless, the 3D simulations based on the commercial code CST STUdio SUITE (which were carried out for the perimeters $\left.l_{\mathrm{x}} / \lambda \leq 10\right)$ demonstrated a similar scenario of the evolution of operating regimes in a coaxial BWO when the transverse size was increased.

For the BWO with an external 2D Bragg synchronizer, the dependence of the oscillation frequency on the electron

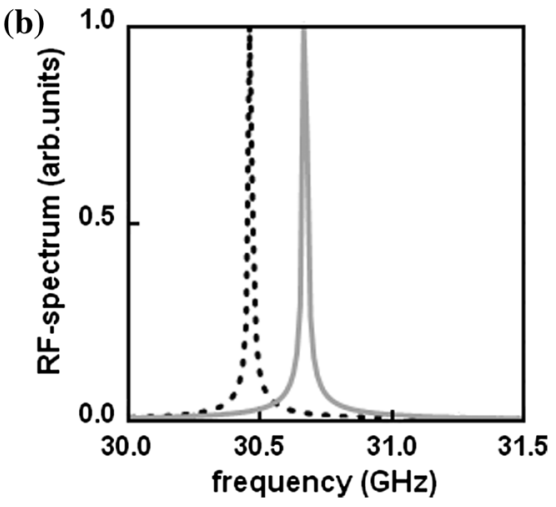

FIG. 2. Simulations of the "freely-oscillating" Ka-band coaxial BWO (without the 2D Bragg synchronizer) of moderate transverse size $l_{\mathrm{x}} / \lambda \sim 4$ and beam current $I_{\text {beam }}=50$ A. (a) Establishment of the stationary oscillation regime at different azimuthal harmonics (which depends on the initial conditions): azimuthally symmetrical mode $m=0$ (black dotted line) and nonsymmetrical mode $m=1$ (gray solid line). (b) The corresponding radiation spectrum. 

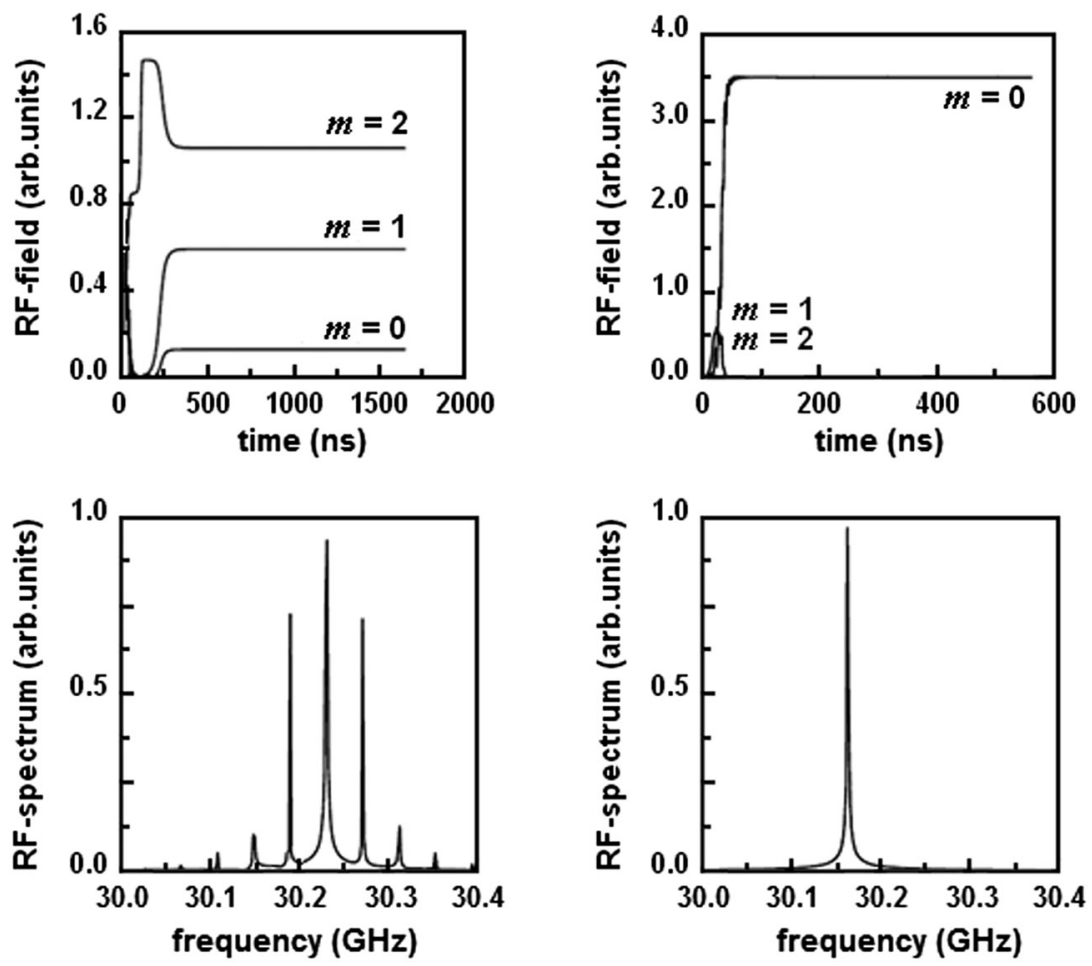

FIG. 3. Simulations of the Ka-band coaxial BWO of large transverse size $l_{\mathrm{x}} / \lambda \sim 16$ with (right column) and without (left column) the 2D Bragg synchronizer: time dependence of the amplitudes of different azimuthal harmonics (top) and radiation spectrum in stationary oscillation regime (bottom) for a moderate beam current $I_{\text {beam }}=50$ A.

energy involves several regions (Fig. 4). Oscillation frequency in these regions belongs to the frequency of different longitudinal modes located inside the Bragg scattering zone of the 2D structure. Frequency shift between these regions of "stabilization frequency" is about $150 \mathrm{MHz}$.

Figure 5 shows the zone on the plane $\left(L_{\mathrm{x}} ; L_{\mathrm{sw}}\right)$, where the single-mode oscillation regime is established for the BWO (a) without and (b) with a 2D Bragg synchronizer. Obviously, the width of such a zone significantly increases when a 2D Bragg synchronizer is installed. Nevertheless, the enhancement of the beam current over the optimal value

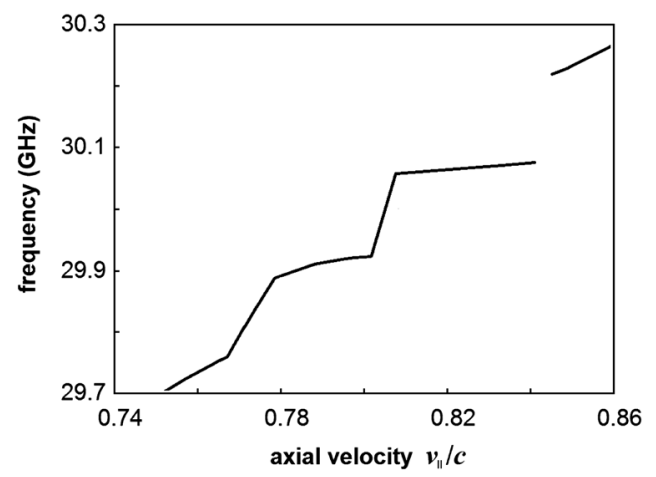

FIG. 4. Dependence of the oscillation frequency on the axial velocity of electrons $v_{\|} / c$ in the coaxial BWO with a $2 \mathrm{D}$ Bragg synchronizer (simulations). leads to the realization of multifrequency regimes which are characterized by the simultaneous excitation of different azimuthal and longitudinal modes (Fig. 6).

It should be noted that the microsecond beam pulse generated by the Saturn accelerator has a slight drift of the mean electron energy. Simulations of the BWO with a 2D Bragg synchronizer demonstrate that such a variation in the beam energy results in broadening of the radiation spectrum [Fig. 7(a)]. Namely, the oscillation frequency shifted, following the beam-energy variations in accordance with Cherenkov synchronism condition (3). In the case of multifrequency oscillations, the broadening of each azimuthal harmonic excited by the beam takes place [Fig. 7(b)].

\section{EXPERIMENTAL SETUP AND RESULTS OF THE EXPERIMENTS}

Experimental study of the Ka-band coaxial BWO was carried out based on the Saturn microsecond accelerator (IAP RAS). A tubular electron beam was formed from a thermionic cathode with a mean diameter of 5 and $0.2 \mathrm{~cm}$ thick (oversize parameter $l_{x} / \lambda \approx 16$ ). The main advantage of the electron beam generated from a thermionic cathode (in comparison with the explosive-emitted beams, which were used in the previous FEM experiments [11-14]), is stability, a more homogeneous distribution of the current over the beam cross section, and that the microsecond pulse duration significantly exceeds the transient time of 

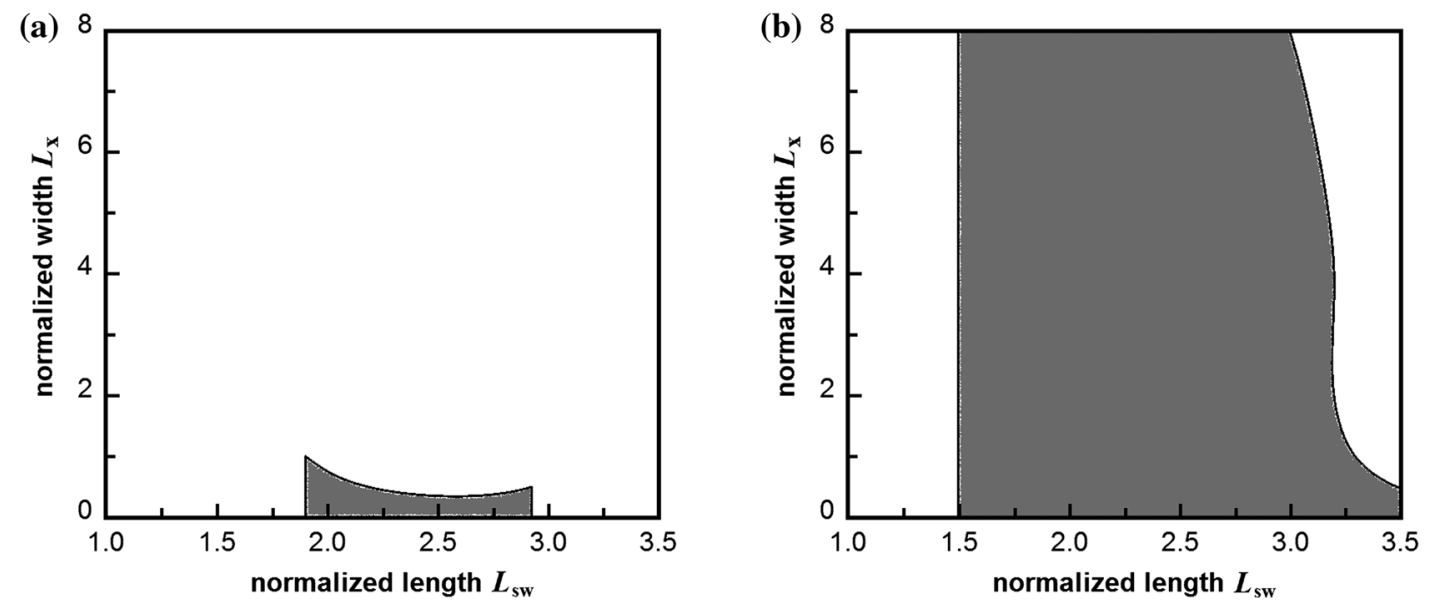

FIG. 5. The zone of establishment of the single-mode oscillation regime in the coaxial BWO (a) without and (b) with a 2D Bragg synchronizer.
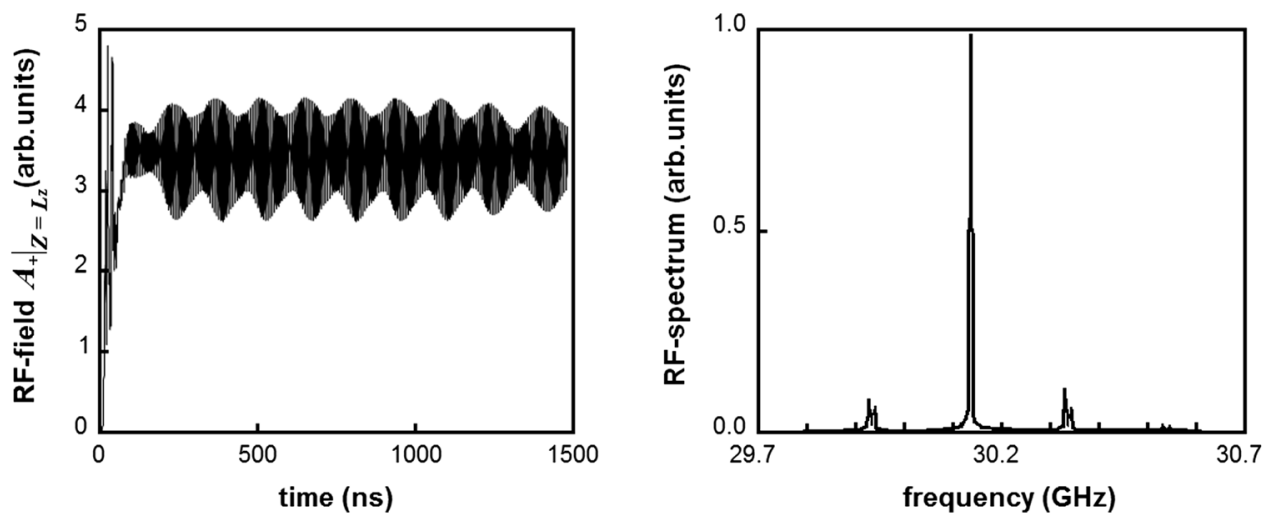

FIG. 6. The results of simulations of the coaxial BWO with a 2D Bragg synchronizer for a high beam current $I_{\text {beam }}=100$ A.
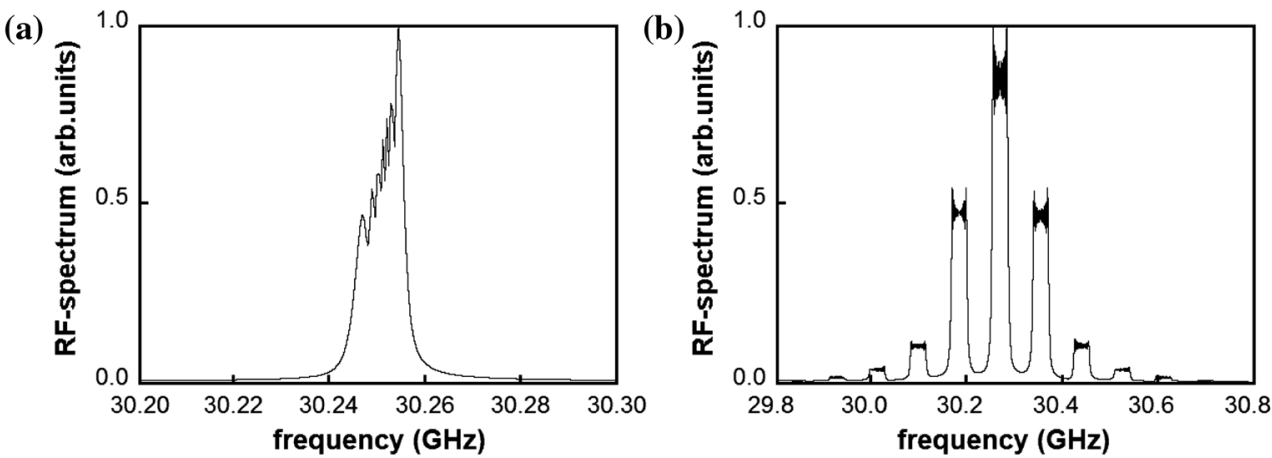

FIG. 7. The results of simulation of the radiation spectrum in the stationary oscillation regime for the coaxial BWO having a small drift of the electron energy on time: (a) with and (b) without a 2D Bragg synchronizer.

oscillations. The electron energies were varied from 200 to $300 \mathrm{keV}$ and the beam current from, 30 to $200 \mathrm{~A}$.

The microwave system with an inner diameter of $4.6 \mathrm{~cm}$ and an outer diameter of $5.5 \mathrm{~cm} \quad\left(r_{0} \approx 2.5 \mathrm{~cm}\right.$ and $a_{0} \approx 0.9 \mathrm{~cm}$ ) was made of oxygen-free copper with high-temperature annealing and exhaustion (Fig. 8), which allows operation with the maximum electric field on the surface, equal to about $250-300 \mathrm{kV} / \mathrm{cm}$. The slow-wave 
structure (section 3 in Fig. 8) was made on the inner conductor with a period of $5.2 \mathrm{~mm}$ and a depth of $1 \mathrm{~mm}$, the length of corrugation was $20 \mathrm{~cm}$. This slow-wave structure provides interaction of the beam with the $\mathrm{TM}_{0,1}$ wave of a coaxial waveguide. To increase the electron-wave coupling coefficient (impedance), the antiphase corrugation of the same period and depth was also made on the outer conductor at a length of $10 \mathrm{~cm}$. The operating $\mathrm{TM}_{0,1}$ wave was reflected from the cathode side of the structure by the cutoff narrowing.

The 2D Bragg structure (section 1 in Fig. 8) $20 \mathrm{~cm}$ long was located on the collector side of the interaction space and separated by a regular waveguide section $2 \mathrm{~cm}$ long
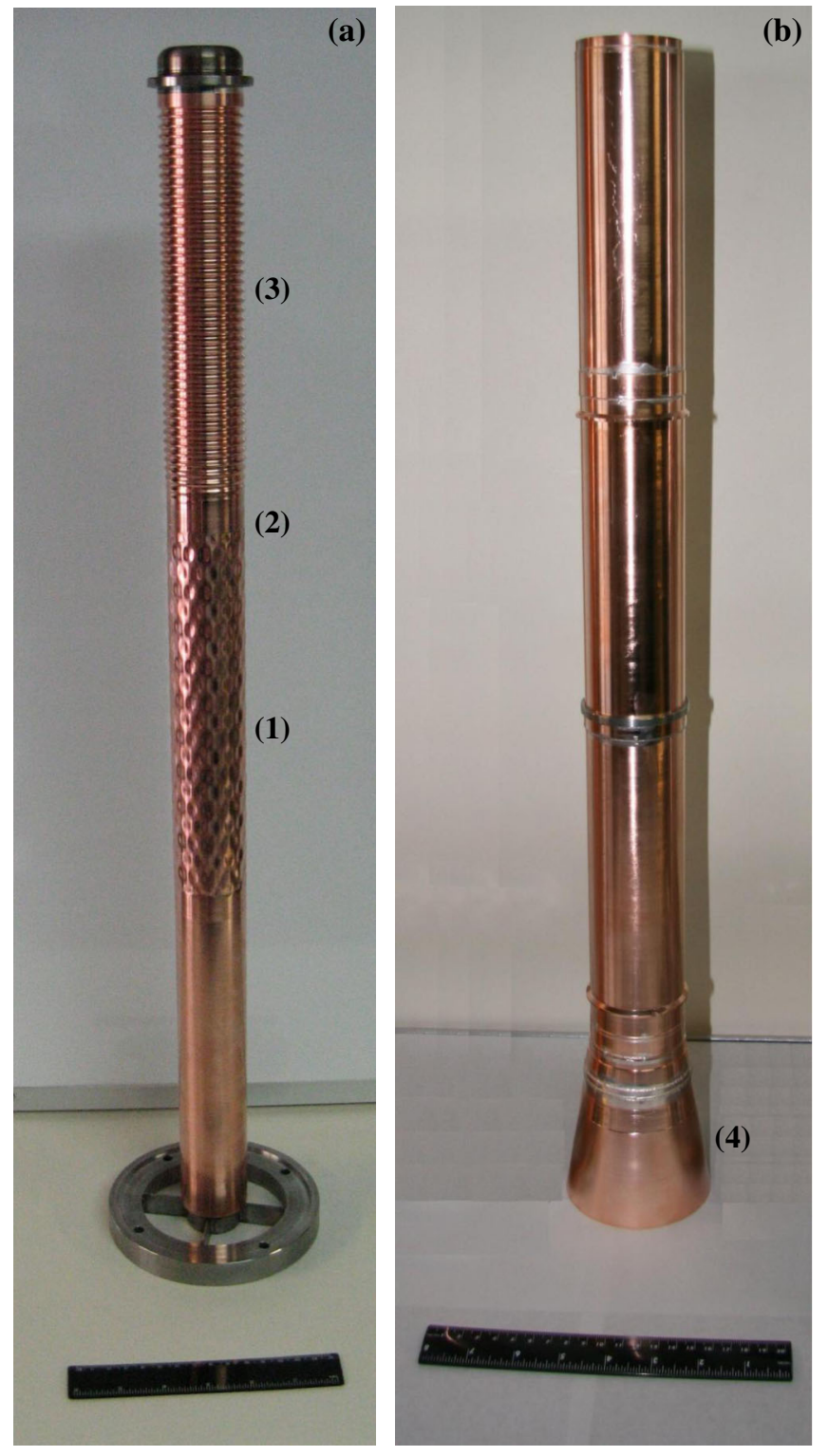

FIG. 8. A photograph of the microwave system of a coaxial Ka-band BWO: (a) inner and (b) outer conductors: (1) 2D Bragg structure, (2) regular waveguide section, (3) slow-wave structure, and (4) output horn. (section 2 in Fig. 8) from the output of the slow-wave structure. The 2D corrugation was machined on the surface of the inner conductor with a period of $2.2 \mathrm{~cm}$, a depth of $0.4 \mathrm{~mm}$, and 16 azimuthal variations (turns). This corrugation provides a feedback loop for two counterpropagating waves of the $\mathrm{TM}_{0,1}$ type ( $\mathbf{A}_{ \pm}$waves) and two azimuthally propagating waves of the $\mathrm{TE}_{16,0}$ type having opposite rotations ( $\mathbf{B}_{ \pm}$waves). Note that simulations of the cavity were conducted within the framework of the average approach described above, as well as using 3D code CST Microwave Studio. Good agreement between the results confirmed the validity of this model.

The microwave parameters of a 2D Bragg structure were examined using a scalar network analyzer. For excitation of the Bragg structures, a wave beam in the form of a $\mathrm{TM}_{0,1}$ wave of a coaxial waveguide was formed at the structure input using an additional transmission line which included four mode converters. These convertors provided the required mode transformation in the operating frequency band: from the launched $\mathrm{TE}_{0,1}$ wave of a single-mode rectangular waveguide to the $\mathrm{TE}_{1,1}$ wave of a circular waveguide (slowly tapered converter No. 1), then to the $\mathrm{TM}_{0,1}$ wave of a circular waveguide (nonadiabatic converter No. 2) and to the TEM wave of a coaxial waveguide (optimized cylindrical insert, converter No. 3), and finally to the $\mathrm{TM}_{0,1}$ wave of a coaxial waveguide (Bragg converter No. 4). An additional coaxial slowly up-tapered waveguide horn $\sim 1 \mathrm{~m}$ long was used to connect from the converters to the oversized coaxial cavity. In accordance with the 3D simulation, the reflection zone for the operating wave in the vicinity of $30.2 \mathrm{GHz}$ with a width of $\sim 0.7-0.8 \mathrm{GHz}$ and a power reflection coefficient of about $60 \%-70 \%$ was measured in the "cold" tests (Fig. 9).
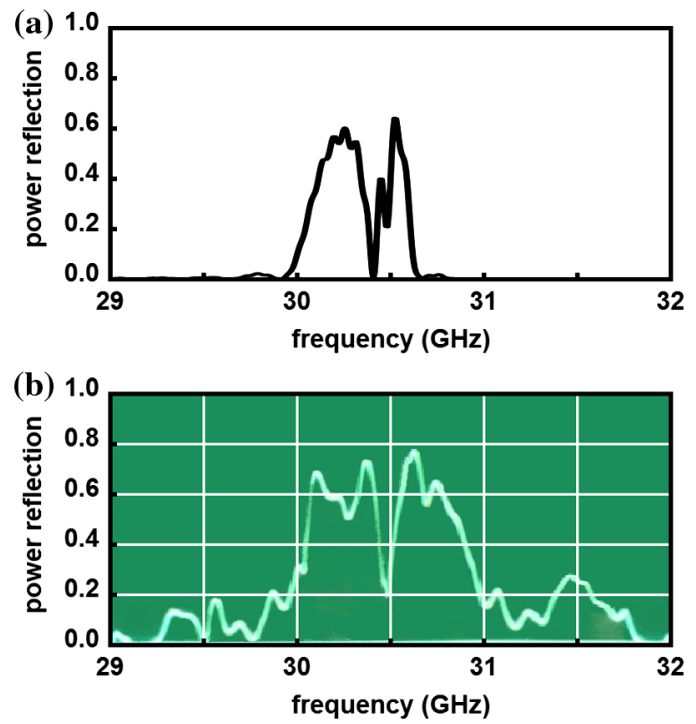

FIG. 9. The results of (a) 3D simulations and (b) "cold" tests of the reflection coefficient of a 2D Bragg structure in the frequency band 29-32 GHz. 
(a)

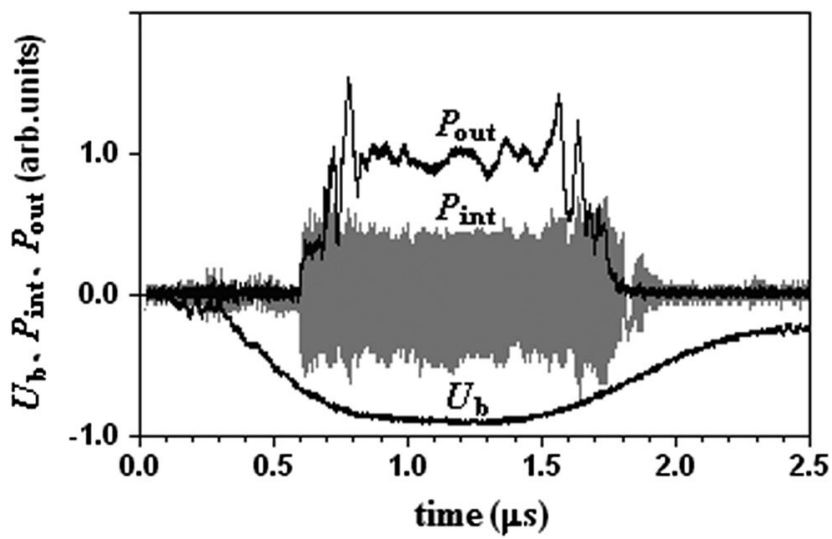

(b)

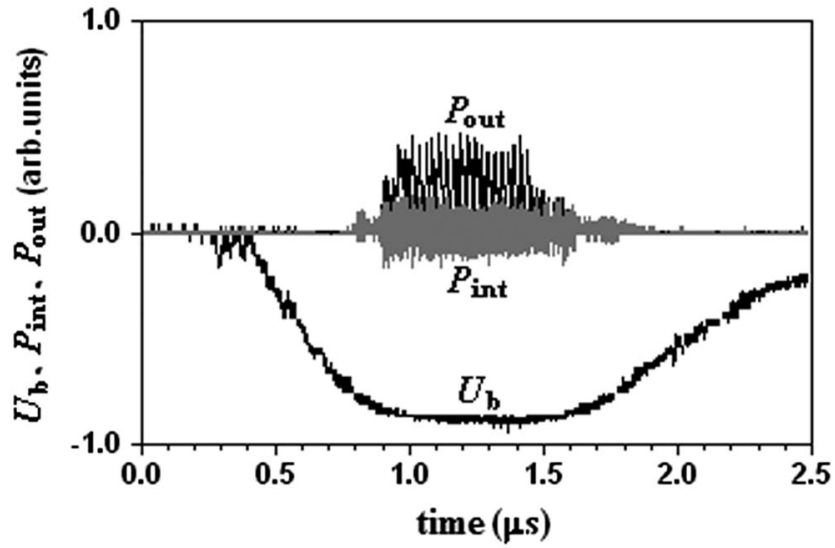

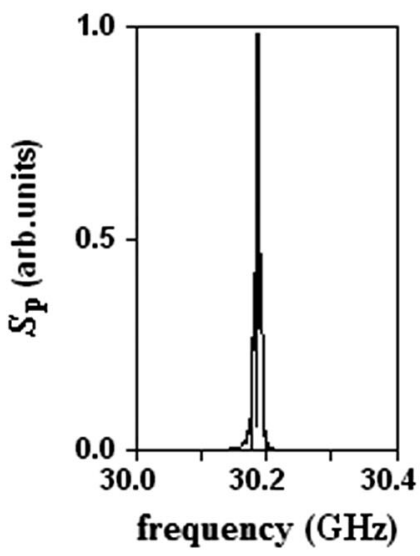

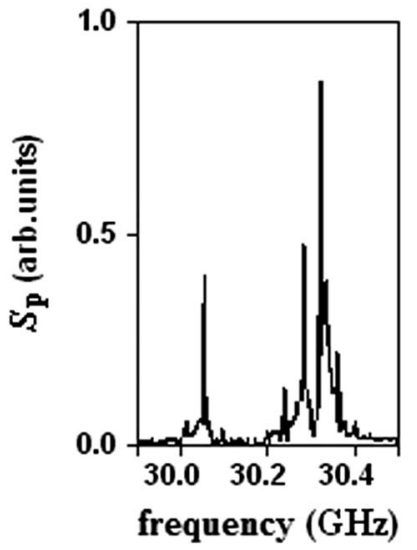

FIG. 10. Typical oscilloscope traces of diode voltage $U_{b}$, rf-pulse $P_{\text {out }}$ and heterodyne signal $P_{\text {int }}$ (left column) and the corresponding frequency spectrum $S_{\mathrm{p}}$ (right column) in Ka-band coaxial BWO with a 2D Bragg synchronizer driven by the Saturn accelerator: (a) narrow-band oscillation regime with the beam parameters $260 \mathrm{keV} / 50 \mathrm{~A}$; (b) multifrequency oscillation regime with the excitation of different azimuthal and longitudinal modes with the beam parameters $200 \mathrm{keV} / 90 \mathrm{~A}$.

The results of experimental studies of the coaxial BWO with a 2D Bragg synchronizer are presented in Fig. 10 and coincide well with the theoretical analysis described above. The starting current of oscillation was estimated at the level 25-30 A. Stable narrow-band oscillation was observed with a beam current of up to 70-80 A. In such regimes, output pulses of microsecond duration and a spectrum width of $\sim 5-10 \mathrm{MHz}$ were registered [Fig. 10(a)]. In accordance with the simulations described above, some broadening of the radiation spectrum is caused by a small drift of the beam energy. In the designed region of parameters, the oscillation frequency belongs to the reflection zone of a 2D Bragg structure near $30.2 \mathrm{GHz}$. We note that several zones of stabilization frequency, each shifted by about 140$150 \mathrm{MHz}$, were observed. Following the simulations given in Fig. 4, this corresponds to the excitation of different longitudinal modes of a 2D Bragg structure. Calorimetric measurement indicates the maximum radiation power at the level 1.5-2 MW in the single-mode regime of oscillation, which corresponds to an electron efficiency of 5\%-10\%. Multifrequency oscillation regimes appear when the beam current exceeds $90 \mathrm{~A}$ and are characterized by a drop of the output power [Fig. 10(b)].

\section{CONCLUSIONS}

Theoretical and experimental studies of a high-power oversized Ka-band BWO of coaxial geometry were carried out. In accordance with simulations, the use of a 2D Bragg synchronizer permits the realization of stable single-mode operation of the oscillator in a wide variation range of the beam current. The oversize factor (perimeter-to-wavelength ratio) amounts to 15-20. As a result, a high potential of the 2D distributed feedback mechanism in the Cherenkov masers driven by spatially extended REBs has been demonstrated.

For the proof-of-principle experiments described here, we chose the scheme of a coaxial BWO where the slowwave structure and the 2D Bragg synchronizer were separated. These experiments were performed based on Saturn, a microsecond accelerator with a thermionicinjection gun. As a result, the electron beam current and the output power were less than in the previous FEM experiments with $2 \mathrm{D}$ distributed feedback, in which explosive-emission accelerators were used [11-14]. The advantage of the oscillators based on thermionic REBs is the high stability of a long-pulse (microsecond) oscillation regime. 
The further increase in the oversize parameter of Cherenkov masers and, thus, radiated power could be achieved by removing the inner conductor and utilizing a hollow cylindrical waveguide with double-period corrugation $[17,18]$. In this scheme, the 2D Bragg structure would be exploited as a slow-wave structure and a high-Q resonator simultaneously. Both schemes have some advantages and disadvantages. Obviously, a single unit scheme is simple for realization from the technological point of view (easy for manufacturing). At the same time, the considered scheme with an external synchronizer is more universal since the Cherenkov BWO can be replaced, for example, by a gyroBWO or a gyro-TWT. Note that the experiments on realization of a single unit scheme of Cherenkov maser in the Ka band is currently in progress at Strathclyde University (Glasgow) with the oversize factor $2 \pi r_{0} / \lambda \approx 6-7$ [22].

\section{ACKNOWLEDGMENTS}

This work was supported in part by the Russian Foundation for Basic Research (projects No. 13-08-01281 and No. 13-08-01075) and the Russian Foundation "Dynasty".-

[1] N. F. Kovalev, M. I. Petelin, M. D. Raizer, A. V. Smorgonsky, and L. E. Tsopp, JETP Lett. 18, 232 (1973).

[2] Y. Carmel, J. Ivers, R. E. Kriebel, and J. A. Nation, Phys. Rev. Lett. 33, 1278 (1974).

[3] V. L. Goldstein, M. Herndon, P. Sprangle, Y. Carmel, and J. A. Nation, Plasma Phys. 17, 23 (1975).

[4] A. S. Elchaninov, F. Ya. Zagulov, S. D. Korovin, G. A. Mesyats, and V. V. Rostov, Tech. Phys. Lett. 6, 1168 (1981).

[5] S. P. Bugaev, V. A. Cherepenin, V. I. Kanavets, A. I. Klimov, A. D. Kopenkin, V. I. Koshelev, V. A. Popov, and A. I. Slepkov, IEEE Trans. Plasma Sci. 18, 525 (1990).

[6] A. N. Vlasov, A. G. Shkvarunets, J. C. Rodgers, Y. Camel, T. M. Jr. Antonsen, T. M. Abuelfadl, D. Lingze, V. A. Cherepenin, G.S. Nusinovich, M. Botton, and V. L. Granatshtein, IEEE Trans. Plasma Sci. 28, 550 (2000).
[7] J. Benford, J. Swegle, and E. Shamiloglu, High Power Microwaves, 2nd ed. (Taylor \& Francis, London, 2007).

[8] V. V. Rostov, M. I. Yalandin, and G. A. Mesyats, IEEE Trans. Plasma Sci. 36, 655 (2008).

[9] N. S. Ginzburg, N. Yu. Peskov, and A. S. Sergeev, Opt. Commun. 112, 151 (1994).

[10] N. S. Ginzburg, N. Yu. Peskov, A. S. Sergeev, A. V. Arzhannikov, and S. L. Sinitsky, Nucl. Instrum. Methods Phys. Res., Sect. A 358, 189 (1995).

[11] A. V. Arzhannikov, N. S. Ginzburg, V. Yu. Zaslavsky, V. G. Ivanenko, I. A. Ivanov, P. V. Kalinin, A. S. Kuznetsov, S. A. Kuznetsov, N. Yu. Peskov, A. S. Sergeev, S. L. Sinitsky, and V. D. Stepanov, JETP Lett. 87, 618 (2008).

[12] A. V. Arzhannikov, A. W. Cross, N. S. Ginzburg, W. He, P. V. Kalinin, I. V. Konoplev, S. A. Kuznetsov, N. Yu. Peskov, A. D. R. Phelps, C. W. Robertson, K. Ronald, A. S. Sergeev, S. L. Sinitsky, V. D. Stepanov, M. Thumm, C. G. Whyte, and V. Yu. Zaslavsky, IEEE Trans. Plasma Sci. 37, 1792 (2009).

[13] I. V. Konoplev, P. McGrane, W. He, A. W. Cross, A. D. R. Phelps, C. G. Whyte, K. Ronald, and C. W. Robertson, Phys. Rev. Lett. 96, 035002 (2006).

[14] I. V. Konoplev, A. W. Cross, A. D. R. Phelps, W. He, K. Ronald, C. G. Whyte, C. W. Robertson, N. S. Ginzburg, N. Yu. Peskov, A. S. Sergeev, V. Yu. Zaslavsky, and M. Thumm, Phys. Rev. E 76, 056406 (2007).

[15] N. S. Ginzburg, N. Yu. Peskov, R. M. Rozental, and A. S. Sergeev, Tech. Phys. Lett. 35, 190 (2009).

[16] N. S. Ginzburg, V. Yu. Zaslavsky, A. M. Malkin, N. Yu. Peskov, and A. S. Sergeev, Tech. Phys. Lett. 36, 83 (2010).

[17] N. S. Ginzburg, A. M. Malkin, A. S. Sergeev, and V. Yu. Zaslavsky, Appl. Phys. Lett. 100, 143510 (2012).

[18] N. S. Ginzburg, A. M. Malkin, A. S. Sergeev, and V. Yu. Zaslavsky, J. Appl. Phys. 113, 104504 (2013).

[19] L. A. Vainshtein, Electromagnetic Waves (Soviet Radio, Moscow, 1957).

[20] A. F. Harvey, Microwave Engineering (Academic Press, New York, 1963).

[21] N. S. Ginzburg, G. S. Nusinovich, and N. A. Zavolsky, Int. J. Electron. 61, 881 (1986).

[22] A. R. Phipps, A. J. MacLachlan, C. W. Robertson, I. V. Konoplev, K. Ronald, A. W. Cross, C. G. Whyte, and A. D. R. Phelps, in Proceedings of the 42 IEEE International Conference on Plasma Science, Belek, Turkey, 2015, p. $2 \mathrm{P} 8$. 